\title{
Tumor hybrid cells: nature and biological significance
}

\author{
Maria S. Tretyakova ${ }^{1}$, Ayalur R. Subbalakshmi ${ }^{2}$, Maxim E. Menyailo ${ }^{1}$, Mohit Kumar Jolly ${ }^{2}$, and \\ Evgeny V. Denisov ${ }^{*}$ \\ ${ }^{1}$ Laboratory of Cancer Progression Biology, Cancer Research Institute, Tomsk National Research \\ Medical Center, Russian Academy of Sciences, Tomsk, Russia \\ ${ }^{2}$ Cancer Systems Biology Laboratory, Centre for BioSystems Science and Engineering, Indian \\ Institute of Science, Bengaluru, India
}

\section{* Correspondence:}

Evgeny V. Denisov

E-mail address: d_evgeniy@oncology.tomsk.ru

\begin{abstract}
Metastasis is the leading cause of cancer death and can be realized through the phenomenon of tumor cell fusion. The fusion of tumor cells with other tumor or normal cells leads to the appearance of tumor hybrid cells (THCs) exhibiting novel properties such as increased proliferation and migration, drug resistance, decreased apoptosis rate and avoiding immune surveillance. Experimental studies showed the association of THCs with a high frequency of cancer metastasis; however, the underlying mechanisms remain unclear. Many other questions also remain to be answered: the role of genetic alterations in tumor cell fusion, the molecular landscape of cells after fusion, the lifetime and fate of different THCs, and the specific markers of THCs, and their correlation with various cancers and clinicopathological parameters. In this review, we discuss the factors and potential mechanisms involved in the occurrence of THCs, the types of THCs, and their role in cancer drug resistance and metastasis, as well as potential therapeutic approaches for the prevention and targeting of tumor cell fusion. In conclusion, we emphasize the current knowledge gaps in the biology of THCs that should be addressed to develop highly effective therapeutics and strategies for metastasis suppression.
\end{abstract}

Keywords: cell fusion, tumor hybrid cell, metastasis, drug resistance

\section{Introduction}

Metastasis accounts for the majority of cancer fatalities and is the least understood stage of tumor progression. The process of metastasis consists of a series of linked and sequential steps: invasion, intravasation, survival during circulation, extravasation, macrometastases, and macrometastases (van Zijl et al., 2011). The underlying mechanisms by which cancer cells acquire the ability to escape the primary tumor site, migrate to distant locations, and reestablish tumorigenesis are not completely known.

According to the "seed and soil" hypothesis, metastasis is governed by interaction and cooperation between the cancer cells (seed) and the host organ (soil) (Akhtar et al., 2019). However, the occurrence and survival of such seed cells is extremely low. Therefore, other mechanisms outside of the "seed and soil" hypothesis may be involved in metastasis.

Cell fusion is a process in which two or more cells fuse and become one due to a common membrane. Cell fusion leads to the formation of hybrid cells with a common genotype of parental cells (LaBerge et al., 2017) but with novel molecular features (Zhang, Huang, et al., 2019). In cancer, cell fusion has been found to play a critical role in metastasis. Hybrid cells derived from cancer cells or cancer and normal cells show increased proliferation and migration, drug resistance, decreased apoptosis rate, and avoiding immune surveillance (Gast et al., 2018; Lartigue et al., 2020; Li et al., 2014). Abnormal division of tumor fusion cells may result in the appearance of polyploid cells (Raslova et al., 2007) contributing to genetic complementation by restoring the loss of gene function and the promotion of the survival of such hybrid cells (Duncan et al., 2009; 
Miroshnychenko et al., 2021). However, to date, there is scarce information about genetic changes driving tumor cell fusion, the life circle of tumor hybrid cells (THCs), and the molecular properties that THCs acquire.

In this review, we discuss the factors and mechanisms involved in the occurrence of THCs, the types of tumor fusion cells, and their role in cancer drug resistance and metastasis, as well as potential therapeutic approaches for the prevention and targeting of tumor cell fusion. In conclusion, we emphasize the current knowledge gaps in the biology of THCs that should be addressed to develop highly effective therapeutics and strategies for metastasis suppression.

\section{THC formation: factors and mechanisms}

The mechanisms governing tumor cell fusion are poorly understood. Under normal conditions, fusion events are rare but increase dramatically in pathological conditions such as tissue injury and inflammation (Davies et al., 2009). The pro-inflammatory cytokine tumor necrosis factor- $\alpha$ (TNF$\alpha$ ) was found to induce the fusion between human M13SV1-Cre breast epithelial cells and human MDA-MB-435-pFDR1 triple-negative breast tumor cells (Weiler et al., 2018). Poor vascularization, resulting in hypoxia and deficient access to nutrients, can be another trigger for tumor cell fusion. For example, the fusion between mesenchymal stromal cells (MSCs) and breast tumor cells is significantly increased in hypoxic conditions (Noubissi et al., 2015).

Cell fusion is promoted by fusogenic proteins. These proteins assemble into unilateral or bilateral complexes, which determine the site of cytoplasmic membrane fusion and overcome the energy barriers. The main fusogens are syncytins, which play a key role in the development of human placental syncytiotrophoblasts (H. Zhu et al., 2020). Syncytin1 (Syn1) and annexin A5 are upregulated in prostate cancer (PC3) and muscle (hMYO) cell cocultures and are involved in tumor cell fusion (Uygur et al., 2019). Syn1 is also upregulated in MCF-7 luminal and MDA-MB-231 triple-negative breast cancer cell lines and about $38 \%$ of breast tumor specimens and facilitates the tumor cell fusion with endothelial cells, while blocking its expression inhibits the formation of THCs (Bjerregaard et al., 2006).

When fusogens are not activated, or the fusion mechanism remains unknown, the actin skeleton can serve as other driver of the occurrence of THCs. Examples include the formation of actin protrusions that promote the fusion of macrophages (Faust et al., 2019) and the fusion of human embryonic kidney cells and embryonic mouse fibroblasts (Chan et al., 2020).

Cell fusion depends on the type of cancer and normal cells, as witnessed in observations in the co-culture of human glioblastoma U87 and U373 cells with MSCs. In particular, more hybrids were formed from U87 cells than from U373 cells. The exact reason for the difference in the formation of hybrids is not yet clear, but may be related to specific gene expression features of these two types of glioblastoma cells (Oliveira et al., 2018). The cell-dependent capacity to form THCs is also seen in breast cancer cell lines. For example, MCF-7 luminal breast cancer cells are more prone to the formation of hybrids than triple-negative breast cancer cells - MDA-MB-231 and SUM159 (Miroshnychenko et al., 2021).

Thus, tumor cell fusion is a regulated process that can be initiated by different external and internal factors (Fig. 1). However, it is unknown what changes occur in the cells before fusion, what signaling pathways are induced by cell fusion drivers, and what processes are activated and suppressed in THCs. These and other questions need to be answered, and a lot of work remains to be done in the future to understand more deeply the mechanisms of THC formation.

\section{Types of THCs}

Tumor cells may fuse both with other tumor cells and different normal cells such as immune cells, MSCs, and fibroblasts (Fig. 2). All these fusion types result in an acquisition of new features and an increase in the aggressiveness of tumor cells.

Fusion between tumor cells. Tumor cells tend to fuse with other tumor cells regardless of the type of cancer. Such fusion can afford tumor cells to change genomic material faster and on a 
much larger scale than genetic mutations. The resulting hybrids of tumor cells acquire new properties such as drug resistance and increased metastatic potential (Searles et al., 2018).

Fusion between tumor and immune cells. Fusion of leukocytes and tumor cells can initiate metastasis. This theory was proposed more than a century ago by Prof. Otto Aichel (Vorträge und Aufsätze über entwickelungsmechanik der Organismen, 1905). Recently, various studies have provided evidence showing that the fusion of cancer cells and leukocytes leads to the formation of metastasis. Leukocyte-tumor cell fusions are frequently observed in lymph node metastases of patients who underwent bone marrow transplantation operations. These hybrids contain a mixture of donor and recipient DNA (Chakraborty et al., 2004; LaBerge et al., 2017; Yilmaz et al., 2005).

Macrophages are divided into two main types: M1 and M2. M1 macrophages are proinflammatory and characterized by the release of inflammatory cytokines. M2-macrophages are considered anti-inflammatory and associated with the release of interleukins (IL)-4, IL-13, and IL10, and promote tumor growth and progression (Nie et al., 2019; Yin et al., 2016; C. Zhu et al., 2017). Fusion between cancer cells and M2 macrophages leads to the formation of hybrids with behavioral properties of the macrophage (Ding et al., 2012) and features of cancer stem cells. These hybrids are characterized by aggressive demeanor, including increased migration, invasion, and tumorigenicity (Ding et al., 2012). Nevertheless, several THCs may undergo apoptosis (Fig. 1) and only some of them survive in the primary tumor, penetrate the circulation, and reach distant sites to form metastases (Miroshnychenko et al., 2021). Interestingly, in distant organs, metastases can be formed both from THCs and tumor cells which get rid of the macrophage part. And other hybrids reaching the target organ gives rise to metastasis, or only its tumor component (LaBerge et al., 2017).

However, the place of fusion of tumor cells and macrophages remains an open question. Does it occur in the blood, the tumor, or in the lymphatic system? The impact of tumor cellmacrophage fusion cells on the immune system is also unclear and is of great interest for future research.

Fusion between tumor cells and MSCs. Tumor cells can merge with MSCs and thus increase their malignant potential (Y. Wang et al., 2012; Wei et al., 2014; Xu et al., 2014; Xue et al., 2015). Tumor cell-MSC fusion during hypoxia promotes the formation of hybrids with increased migration ability and stem features that may enable their dissemination to distant sites (Noubissi et al., 2015). Several studies in vitro and in vivo showed that MSCs fuse with breast tumor cells and novel hybrids possess more aggressive properties than the parental tumor cells (C. Melzer et al., 2018, 2018, 2019). The fusion of MSCs and breast cancer cells contributes to elevated tumor cell plasticity and heterogeneity in tumorigenic potential and chemotherapeutic responsiveness (Catharina Melzer et al., 2021).

Fusion between tumor cells and fibroblasts. Fibroblasts represent the largest population of cells in the tumor environment (Truffi et al., 2020). Cancer-associated fibroblasts are a heterogeneous and highly plastic group of cells which are one of the key players in the regulation of metastatic cascade (Suetsugu et al., 2021). Fusion between fibroblasts and tumor cells is considered to be a part of the tumor-stroma interaction (Vucicevic Boras et al., 2018). Cancerassociated fibroblasts fuse with prostate tumor cells and form hybrids in coculture model. Most of the resulting tumor fusion cells die, but the surviving hybrids have increased proliferative activity and aggressiveness (R. Wang et al., 2012). Moreover, the survived hybrids acquire genomic alterations and novel molecular characteristics, which can be the potential cause of tumor invasion and metastasis (R. Wang et al., 2012).

Thus, fusion with different types of cells can be a mechanism of the evolution of cancer cells by gaining novel properties to survive under unfavorable hypoxic conditions and pressure from the immune system.

\section{Role of THCs in metastasis}

Various manifestations of cancer cells forming hybrid cells together with stromal cells have been reported (Table 1). For instance, human mesenchymal stroma/stem-like cells (MSCs) can 
spontaneously fuse with MDA-MB-231 breast cancer during co-culture to form two different aneuploid populations with varying short tandem repeat (STR) profiles (MDA-hyb1, MDA-hyb2). Both these populations had higher proliferation rate than MDA-MB-231 cells, and enrichment of mesenchymal markers such as FN1, SNAI2 and MMP9. In vivo experiments revealed higher tumorigenicity and metastatic potential of both the hybrid cell populations (C. Melzer et al., 2018). Another study reported the fusion of human mesenchymal stromal cells with MCF-7 and T74D breast tumor cells (Chitwood et al., 2018), both of which are more epithelial cells as compared to MDA-MB-231 (George et al., 2017). Spontaneous cell fusion can also happen in vivo, and lung metastases were shown to possess a higher frequency of hybrid cells formed between MSCs and murine fat pad tumor cells (PyVT) when compared to primary breast tumor, suggesting that hybrid cells can form in primary tumor, metastasize and then proliferate at the metastatic site (Chitwood et al., 2018). Similar traits are reported on fusion of mesenchymal cells with those of same (mesenchymal) lineage too. The fusion of fibroblasts IMR90 with precancerous cells of the same lineage (IMR90 E6/E7) led to genetically unstable clones, each of which was more aggressive and metastatic as compared to their parental populations. Reinforcing observations were made upon sarcoma cell fusion hybrids. When engrafted in mice, hybrid cells formed tumors reminiscent of undifferentiated pleomorphic sarcoma (UPS) in terms of genetics, clinical behavior, and tumor histology (Lartigue et al., 2020). Put together, these observations highlight the increased metastatic potential of tumor hybrid cells.

Higher heterogeneity has been proposed to underlie this metastatic propensity of hybrid cells (Gast et al., 2018). Hybrid cells formed in vitro by the fusion of bone marrow derived macrophages with murine cells MC38 (intestinal epithelial cancer) and B16F10 (melanoma) possessed a fused nuclei exhibiting neoplastic transcriptional identity, while notably, retained macrophage gene expression signatures. They also exhibit contact inhibition and mesenchymal histologic sheet-like features. In vivo, hybrid cells are a rare subpopulation (0.03-0.69\%), but have a shorter doubling time and enhanced metastatic ability. In patients, circulating hybrid cells (CHCs) were identified based on co-expression of CD45 (leukocyte marker) with CK (cytokeratin: epithelial marker). The frequency of CHCs, but not that of CTCs (circulating tumor cells: CD45-negative, CK-positive), was observed to be a prognostic marker for patient survival, regardless of disease stage. Intriguingly, fused cells demonstrated a combination of adhesion biases, as identified by a microenvironment microarray (MEMA) platform. Such broader adhesive affinity may offer survival advantage during metastatic cascade, although direct experimental validation of this hypothesis remains to be yet established.

Another aspect of metastatic fitness observed in tumor hybrid cells is the acquisition of EMT and/or stemness, two interrelated traits that can accelerate metastasis (Celià-Terrassa et al., 2020). MCF-7 cells, when fused with macrophages, had upregulated Snail1, Snail2, and Vimentin, and decreased E-cadherin levels (Ding et al., 2012). Upon fusion with macrophages, both MCF-7 and MDA-MB-231 cells had a higher percentage of CD44+/ CD24- cells and enhanced tumorigenicity in vivo, suggesting that cell fusion can also be a source of cancer stem cells (CSCs). Similar observations are reported in fusion of lung cancer cells (Xu et al., 2014; Zhang, Kong, et al., 2019) and gastric epithelial cells (He et al., 2015). Interestingly, the traits of MCF-7/ macrophage hybrid cells, such as expression of M2 macrophage marker CD163, could not be explained by paracrine engagement of MCF-7 cells with macrophages (Shabo et al., 2015). Further, breast cancer patients with $>25 \%$ of cancer cells expressing CD163 had a worse disease free and recurrence free survival relative to those with $<25 \%$ of such cells. Besides displaying EMT and stemness, THCs can also be immune-evasive and their frequency in circulation can indicate metastatic ability of a tumor (Aguirre et al., 2020).

The abovementioned studies offer putative mechanistic insights into earlier phenomenological observations on increased aggressive behavior of somatic cell fusion events, as reported in many cancers (De Baetselier et al., 1984; J. M. Pawelek, 2000; John M. Pawelek et al., 2008; Sidebottom et al., 1983; Sieler et al., 2021). Cell-cell fusion can lead to DNA exchange 
(Searles et al., 2018), which can drive aneuploidy and increased genetic and phenotypic heterogeneity, thus aggravating cancer progression.

\section{Role of THCs in drug resistance}

Besides mediating metastasis, THCs can also drive resistance against various drugs. In a metastatic colon carcinoma model in vivo (Carloni et al., 2013), fused cells were found to be resistance to both 5-flourouracil (5-FU) and oxaliplatin. Similarly, hybrid cells formed due to fusion of two sister subpopulations - 168FAR and 44FTO - was found to be more resistant to both melphalan and methotrexate than either parental subpopulation. Also, these two co-existing subpopulations had varied organotrophic behavior; the hybrid clone showed spontaneous metastatic traits as well (Miller et al., 1989). Beyond chemoresistance, spontaneous fusion between M2-macrophages and MCF-7 cancer cells can drive radioresistance too in vitro; such hybrid cells have enhanced DNA repair capacity and less heterogeneity in DNA damage upon exposure to radiation (Lindström et al., 2017). While in vivo existence of these radioresistance traits remain to be observed, intriguingly, radiation itself can drive homotypic cell fusion of a subpopulation of glioblastoma cells (Kaur et al., 2015). These pre-existing radiation resistant mononucleated cells can survive radiotherapy through undergoing a cell cycle arrest and repairing their damaged DNA. They undergo cell-cell fusion to form multinucleated and giant cells (MNGCs).

EMT and/or stemness have been often associated with resistance to many chemotherapeutic drugs, radiation therapy, targeted therapy and immunotherapy across multiple cancers (Dongre et al., 2017; Mal et al., 2020; S. Sahoo et al., 2021; Sarthak Sahoo et al., 2021; P. Zhang et al., 2014; Zheng et al., 2015), but whether these processes are responsible for aggressive behavior of THCs remains to be elucidated. Further analysis of any causal contribution of these processes can be performed through dissecting the heterogeneity of THCs seen in primary tumor and/or circulation. For instance, a comparison of hybrid clone cells formed by spontaneous fusion events of human M13SV1-EGFP-Neo breast epithelial cells and HS578T-Hyg, MDA-MB-435-Hyg and MDAMB-231-Hyg cancer cells demonstrated that fusion cells formed by HS578 cells had stronger in vitro tumor-initiating traits as compared to those formed by MDA-MB-231 and MDA-MB-453 cells, as identified by surface marker (frequency of ALDH1+ cells) and functional (mammosphere) assays (Fahlbusch et al., 2020). Similarly, hybrid cells in circulation were shown to reflect the heterogeneity of both epithelial and non-epithelial malignancies, and can be used as a translational non-invasive readout of tumor aggressiveness, given their higher frequency than that of CTCs (Dietz et al., 2021). Hybrid clones can also be metabolically heterogeneous, as noted for subpopulations of CSCs with varying EMT phenotypes (Luo et al., 2018), but overall, they display an enriched Warburg-like phenotype (upregulated glycolysis) (Brito et al., 2021). Cell-to-cell heterogeneity has been shown to accelerate tumor progression and/or metastasis in various contexts, such as cooperation between EMT and non-EMT cells (Neelakantan et al., 2017; Tsuji et al., 2008), but whether such cooperation is seen among tumor hybrid cells and whether any such non-cell autonomous behavior has a functional role to play in enhancing metastasis remains to be identified in vitro and in vivo.

It should be noted that not every instance of cell fusion necessarily implies higher metastatic and/or chemoresistant set of features. For example, MDA-hyb3 cells formed by in vivo spontaneous fusion of MSCs with MDA-MB-231 cells had reduced tumor-forming and metastatic ability compared to MDA-MB-231 cells, despite having enhanced proliferation (C. Melzer et al., 2019). Similar observations were noted for SK-MSC-hyb1 and -hyb2 ovarian cancer hybrids (fusion of SK-OV-3 human ovarian cells with MSCs) (C. Melzer et al., 2020). A detailed molecular mapping of such less metastatic hybrid cells remains to be done. Therefore, a comparative analysis of more vs. less metastatic and aggressive hybrid clones will be essential to identify promising therapeutic vulnerabilities that may be clinically relevant.

\section{Therapeutic options for targeting THCs}


The prevention of tumor cell fusion and targeting THCs seem to be an attractive approach for anticancer treatment, particularly a decrease in therapy resistance and the suppression of metastasis. Basically, two main approaches can be distinguished to affect neoplastic and nonneoplastic components using different molecules as potential therapeutic targets (Fig. 3).

Matrix metalloproteinase MMP9 is a critical molecule for the TNF- $\alpha$-induced fusion of breast epithelial (M13SV1) and triple-negative breast tumor cells (MDA-MB-435) (Weiler et al., 2018). The inhibition of MMP9 by SB-3CT leads to a decreased fusion rate of these cells (Saito et al., 2015). Likewise, tetracycline-based antibiotic minocycline is effective to target MMP9 and block the TNF- $\alpha$-induced fusion frequency of M13SV1 and MDA-MB-435 cells (Weiler et al., 2018).

Toll-like receptors (TLRs) belong to the group of pathogen recognition receptors which play a crucial role in the innate immune system. TLR4-mediated signaling is implicated in tumor cell invasion, survival, and metastasis in various cancers (Yang et al., 2014). TLR4 and TLR9 are highly expressed in M13MDA-435-1 and -3 hybrid breast tumor cells (Fried et al., 2016), while their cultivation in the presence of the TLR4 ligand lipopolysaccharide induces apoptosis in all hybrid clones (Fried et al., 2016).

The activation of the Wnt/ $\beta$-catenin signaling pathway is crucial for the artificial fusion between breast tumor cells (N2O2) and macrophages using polyethylene glycol hydrogels and the promotion of proliferation, migration, invasion, and colony formation of THCs (Zhang, Huang, et al., 2019). In turn, XAV-939, a small molecule inhibitor of the $\mathrm{Wnt} / \beta$-catenin signaling pathway, is able to reduce the capability of fusion of macrophages with tumor cells leading to a decrease in cell proliferation, migration, and invasion (Zhang, Huang, et al., 2019).

Cell fusion modifies epigenetic landscape unlocking the expression of transcription factors, for example, Nanog, which is directly involved in cell reprogramming. It was shown that Nanog is strongly expressed by spontaneous mesenchymal hybrid cells generated from sarcoma cells (B105-DsRed, IB106-GFP, and IB105/106) and fibroblasts (IMR90) (Lartigue et al., 2020; Merle et al., 2021). Inhibition of Nanog by siRNA significantly decreases the migration capacity of THCs (Merle et al., 2021).

Tumor cell-macrophage hybrids express both macrophage (CD14, CD68, CD163, CD204, and CD206) and tumor-specific markers (ALCAM, MLANA, KRT, EpCAM, CXCR4, and CD44) (Clawson et al., 2015). These fusion cells also express integrin subunits $(\alpha 3, \alpha 5, \alpha 6, \alpha v$, $\beta 1$, and $\beta 3$ ) and GnT-V ( $\beta 1,6$-acetylglucosaminyltransferase-V) (John M. Pawelek, 2005). Therefore, the aforementioned molecules can be potential targets for destroying tumor-macrophage fusion cells (Soltantoyeh et al., 2021). Targeting GnT-V, as well as SPARC, SNAIL, and MITF, in combination with chimeric antigen receptor (CAR) $\mathrm{T}$ cells that are specific to surface proteins ( $\beta 1,6$-branched oligosaccharides, MET, and LAMP1) of tumor-macrophage fusion cells, may improve chimeric antigen receptor CAR T cell performance in metastatic melanoma (Soltantoyeh et al., 2021).

THCs themselves can act as anti-tumor therapeutic options. Purified dendritic cell-tumor fusion hybrids supplemented with the non-adherent dendritic cell population elicit the powerful antitumor immune response in breast cancer model in vitro (Y. Zhang et al., 2014). Similarly, fusion between dendritic cells and cancer cells generates hybrids that activate both $\mathrm{CD}^{+}$and $\mathrm{CD} 8^{+}$ $\mathrm{T}$ cells, therefore activating the antitumor immunity (S. Koido et al., 2014; Shigeo Koido et al., 2014). These results indicate that fusion cell vaccines can be an effective instrument to induce antigen-specific responses and to activate anti-tumor immunity; however, further research is needed to evaluate this option in in vivo models.

Thus, different therapeutic options can be addressed both to prevent tumor cell fusion and to destroy THCs. However, the efficiency of all of them was demonstrated in vitro and further studies on in vivo and clinical models are required especially in terms of understanding the mechanisms of THC formation in the multilevel organism systems and the development of instruments for their control. 


\section{Challenges and current trends}

Cell fusion promotes tumor growth and progression through the formation of new cells with increased drug-resistance, immunotolerance, and metastatic properties than the parental or unfused cells. Therefore, the identification of THCs and revealing their molecular features may be effective in developing therapeutic approaches against cancer metastasis. Despite the abundance of studies devoted to THCs, several critical points remain to be elucidated.

The problem of identifying THCs remains unresolved. At present, THCs are identified focusing on the specific markers of parental cells. However, fusion cells that lack those markers cannot be categorized as THCs. This challenge can possibly be overcome through achieving a deep understanding of underlying cell/molecular biology of different types of THCs. This task can be difficult because of the same or largely overlapping genetic content of parental cells and THCs.

Another unresolved problem is related to the absence of information on the specificity of THCs to various cancers. THCs can be metastatic and aggressive to varying degrees, but it is unknown whether the frequency and spectrum of THCs correlates with clinicopathological parameters. Identification of specific markers for the identifying THCs can help answer this too.

The current data indicate the strong heterogeneity of THCs which results in the presence of cell populations with varying degrees of drug resistance, immune tolerance, and invasive and metastatic potentials. This evidence together with a diversity of tumor cells significantly increases the level of intra- and inter-tumor heterogeneity and thus the chances of treatment failure and poor outcome. Therefore, deciphering cell heterogeneity and identifying the most aggressive cell populations is one of the key aims to uncover the nature of THCs. In this case, the potential instrument can be the use of approaches of single-cell genomics and proteomics which also can shed light on the discovery of markers specific to different types of THCs.

The questions about genetic alterations that can trigger tumor cell fusion, the molecular landscape of cells after fusion, and the lifetime and fate of different THCs also remain unanswered.

Thus, further research should focus on a comprehensive and multi-omic analysis of THCs to reveal genetic alterations leading to tumor cell fusion and specific markers of THCs, to investigate how strong the molecular landscape of hybrid cells changes as compared to parental cells, and to understand the specificity of THCs to various cancer types and their correlation with clinical and pathological parameters.

\section{Funding}

This research was funded by the Russian Science Foundation (grant \#19-75-30016). MKJ was supported by Ramanujan Fellowship awarded by Science and Engineering Research Board (SERB), Department of Science and Technology (DST), Government of India (SB/S2/RJN 049/2018).

\section{Acknowledgement}

We acknowledge Mr. Atchuta Srinivas Duddu (IISc Bangalore) for help with artwork for figures.

\section{Conflict of Interest}

The authors declare no conflict of interest. 


\section{References}

Aguirre, L. A., Montalbán-Hernández, K., Avendaño-Ortiz, J., Marín, E., Lozano, R., et al. (2020). Tumor stem cells fuse with monocytes to form highly invasive tumor-hybrid cells. Oncoimmunology, 9, 1773204. doi:10.1080/2162402x.2020.1773204

Akhtar, M., Haider, A., Rashid, S., \& Al-Nabet, A. (2019). Paget's "Seed and Soil" Theory of Cancer Metastasis: An Idea Whose Time has Come. Adv Anat Pathol, 26, 69-74. doi:10.1097/pap.0000000000000219

Bjerregaard, B., Holck, S., Christensen, I., \& Larsson, L.-I. (2006). Syncytin is involved in breast cancer-endothelial cell fusions. Cellular and Molecular Life Sciences CMLS, 63, 19061911.

Brito, A., Merle, C., Lagarde, P., Faustin, B., Devin, A., et al. (2021). Cell fusion enhances energy metabolism of mesenchymal tumor hybrid cells to sustain their proliferation and invasion. BMC Cancer, 21, 863. doi:10.1186/s12885-021-08561-6

Carloni, V., Mazzocca, A., Mello, T., Galli, A., \& Capaccioli, S. (2013). Cell fusion promotes chemoresistance in metastatic colon carcinoma. Oncogene, 32, 2649-2660. doi:10.1038/onc.2012.268

Celià-Terrassa, T., \& Jolly, M. K. (2020). Cancer Stem Cells and Epithelial-to-Mesenchymal Transition in Cancer Metastasis. Cold Spring Harbor Perspectives in Medicine, 10. doi:10.1101/cshperspect.a036905

Chakraborty, A., Lazova, R., Davies, S., Bäckvall, H., Ponten, F., et al. (2004). Donor DNA in a renal cell carcinoma metastasis from a bone marrow transplant recipient. Bone Marrow Transplant, 34, 183-186. doi:10.1038/sj.bmt.1704547

Chan, K. M. C., Son, S., Schmid, E. M., \& Fletcher, D. A. (2020). A viral fusogen hijacks the actin cytoskeleton to drive cell-cell fusion. Elife, 9. doi:10.7554/eLife.51358

Chitwood, C. A., Dietzsch, C., Jacobs, G., McArdle, T., Freeman, B. T., et al. (2018). Breast tumor cell hybrids form spontaneously in vivo and contribute to breast tumor metastases. APL Bioeng, 2, 031907. doi:10.1063/1.5024744

Clawson, G. A., Matters, G. L., Xin, P., Imamura-Kawasawa, Y., Du, Z., et al. (2015). Macrophage-tumor cell fusions from peripheral blood of melanoma patients. PloS One, 10, e0134320. doi:10.1371/journal.pone.0134320

Davies, P. S., Powell, A. E., Swain, J. R., \& Wong, M. H. (2009). Inflammation and proliferation act together to mediate intestinal cell fusion. PLoS One, 4, e6530. doi:10.1371/journal.pone.0006530

De Baetselier, P., Roos, E., Brys, L., Remels, L., Gobert, M., et al. (1984). Nonmetastatic tumor cells acquire metastatic properties following somatic hybridization with normal cells. Cancer and Metastasis Reviews, 3, 5-24. doi:10.1007/bf00047690

Dietz, M. S., Sutton, T. L., Walker, B. S., Gast, C. E., Zarour, L., et al. (2021). Relevance of circulating hybrid cells as a non-invasive biomarker for myriad solid tumors. Scientific Reports, 11, 13630. doi:10.1038/s41598-021-93053-7

Ding, J., Jin, W., Chen, C., Shao, Z., \& Wu, J. (2012). Tumor associated macrophage $\times$ cancer cell hybrids may acquire cancer stem cell properties in breast cancer. PLoS One, 7, e41942. doi:10.1371/journal.pone.0041942

Dongre, A., Rashidian, M., Reinhardt, F., Bagnato, A., Keckesova, Z., et al. (2017). Epithelialto-Mesenchymal Transition Contributes to Immunosuppression in Breast Carcinomas. Cancer Res, 77, 3982-3989. doi:10.1158/0008-5472.can-16-3292

Duncan, A. W., Hickey, R. D., Paulk, N. K., Culberson, A. J., Olson, S. B., et al. (2009). Ploidy reductions in murine fusion-derived hepatocytes. PLoS Genet, 5, e1000385. doi:10.1371/journal.pgen.1000385

Fahlbusch, S. S., Keil, S., Epplen, J. T., Zänker, K. S., \& Dittmar, T. (2020). Comparison of hybrid clones derived from human breast epithelial cells and three different cancer cell lines regarding in vitro cancer stem/ initiating cell properties. BMC Cancer, 20, 446. doi:10.1186/s12885-020-06952-9 
Faust, J. J., Balabiyev, A., Heddleston, J. M., Podolnikova, N. P., Baluch, D. P., et al. (2019). An actin-based protrusion originating from a podosome-enriched region initiates macrophage fusion. Mol Biol Cell, 30, 2254-2267. doi:10.1091/mbc.E19-01-0009

Fried, S., Tosun, S., Troost, G., Keil, S., Zaenker, K. S., et al. (2016). Lipopolysaccharide (LPS) Promotes Apoptosis in Human Breast Epithelial $\times$ Breast Cancer Hybrids, but Not in Parental Cells. PloS One, 11, e0148438. doi:10.1371/journal.pone.0148438

Gast, C. E., Silk, A. D., Zarour, L., Riegler, L., Burkhart, J. G., et al. (2018). Cell fusion potentiates tumor heterogeneity and reveals circulating hybrid cells that correlate with stage and survival. Sci $A d v$, 4, eaat7828. doi:10.1126/sciadv.aat7828

George, J. T., Jolly, M. K., Xu, S., Somarelli, J. A., \& Levine, H. (2017). Survival Outcomes in Cancer Patients Predicted by a Partial EMT Gene Expression Scoring Metric. Cancer Research, 77, 6415-6428. doi:10.1158/0008-5472.can-16-3521

He, X., Li, B., Shao, Y., Zhao, N., Hsu, Y., et al. (2015). Cell fusion between gastric epithelial cells and mesenchymal stem cells results in epithelial-to-mesenchymal transition and malignant transformation. BMC Cancer, 15, 24. doi:10.1186/s12885-015-1027-1

Kaur, E., Rajendra, J., Jadhav, S., Shridhar, E., Goda, J. S., et al. (2015). Radiation-induced homotypic cell fusions of innately resistant glioblastoma cells mediate their sustained survival and recurrence. Carcinogenesis, 36, 685-695. doi:10.1093/carcin/bgv050

Koido, S., Enomoto, Y., Apostolopoulos, V., \& Gong, J. (2014). Tumor regression by CD4 Tcells primed with dendritic/tumor fusion cell vaccines. Anticancer Research, 34, 39173924.

Koido, S., Homma, S., Kan, S., Takakura, K., Namiki, Y., et al. (2014). Induction of antigenspecific cytotoxic T lymphocytes by fusion cells generated from allogeneic plasmacytoid dendritic and tumor cells Corrigendum in/10.3892/ijo. 2015.2954. International Journal of Oncology, 45, 470-478.

LaBerge, G. S., Duvall, E., Grasmick, Z., Haedicke, K., \& Pawelek, J. (2017). A Melanoma Lymph Node Metastasis with a Donor-Patient Hybrid Genome following Bone Marrow Transplantation: A Second Case of Leucocyte-Tumor Cell Hybridization in Cancer Metastasis. PLoS One, 12, e0168581. doi:10.1371/journal.pone.0168581

Lartigue, L., Merle, C., Lagarde, P., Delespaul, L., Lesluyes, T., et al. (2020). Genome remodeling upon mesenchymal tumor cell fusion contributes to tumor progression and metastatic spread. Oncogene, 39, 4198-4211.

Li, H., Feng, Z., Tsang, T. C., Tang, T., Jia, X., et al. (2014). Fusion of HepG2 cells with mesenchymal stem cells increases cancer-associated and malignant properties: an in vivo metastasis model. Oncol Rep, 32, 539-547. doi:10.3892/or.2014.3264

Lindström, A., Midtbö, K., Arnesson, L. G., Garvin, S., \& Shabo, I. (2017). Fusion between M2macrophages and cancer cells results in a subpopulation of radioresistant cells with enhanced DNA-repair capacity. Oncotarget, 8, 51370-51386.

doi:10.18632/oncotarget.17986

Luo, M., Shang, L., Brooks, M. D., Jiagge, E., Zhu, Y., et al. (2018). Targeting Breast Cancer Stem Cell State Equilibrium through Modulation of Redox Signaling. Cell Metabolism, 28, 69-86.e66. doi:10.1016/j.cmet.2018.06.006

Mal, A., Bukhari, A. B., Singh, R. K., Kapoor, A., Barai, A., et al. (2020). EpCAM-Mediated Cellular Plasticity Promotes Radiation Resistance and Metastasis in Breast Cancer. Front Cell Dev Biol, 8, 597673. doi:10.3389/fcell.2020.597673

Melzer, C., Ohe, J. V., \& Hass, R. (2020). Altered Tumor Plasticity after Different Cancer Cell Fusions with MSC. Int J Mol Sci, 21. doi:10.3390/ijms21218347

Melzer, C., von der Ohe, J., \& Hass, R. (2018). Enhanced metastatic capacity of breast cancer cells after interaction and hybrid formation with mesenchymal stroma/stem cells (MSC). Cell Commun Signal, 16, 2. doi:10.1186/s12964-018-0215-4 
Melzer, C., von der Ohe, J., \& Hass, R. (2018). In Vitro Fusion of Normal and Neoplastic Breast Epithelial Cells with Human Mesenchymal Stroma/Stem Cells Partially Involves Tumor Necrosis Factor Receptor Signaling. Stem Cells, 36, 977-989. doi:10.1002/stem.2819

Melzer, C., von der Ohe, J., \& Hass, R. (2019). In Vivo Cell Fusion between Mesenchymal Stroma/Stem-Like Cells and Breast Cancer Cells. Cancers (Basel), 11. doi:10.3390/cancers11020185

Melzer, C., von der Ohe, J., Luo, T., \& Hass, R. (2021). Spontaneous fusion of MSC with breast cancer cells can generate tumor dormancy. International Journal of Molecular Sciences, 22, 5930 .

Merle, C., Lagarde, P., Lartigue, L., \& Chibon, F. (2021). Acquisition of cancer stem cell capacities after spontaneous cell fusion. BMC Cancer, 21, 241. doi:10.1186/s12885-02107979-2

Merle, C., Lagarde, P., Lartigue, L., \& Chibon, F. (2021). Acquisition of cancer stem cell capacities after spontaneous cell fusion. BMC Cancer, 21, 1-9.

Miller, F. R., Mohamed, A. N., \& McEachern, D. (1989). Production of a More Aggressive Tumor Cell Variant by Spontaneous Fusion of Two Mouse Tumor Subpopulations. Cancer Research, 49, 4316-4321.

Miroshnychenko, D., Baratchart, E., Ferrall-Fairbanks, M. C., Velde, R. V., Laurie, M. A., et al. (2021). Spontaneous cell fusions as a mechanism of parasexual recombination in tumour cell populations. Nat Ecol Evol, 5, 379-391. doi:10.1038/s41559-020-01367-y

Neelakantan, D., Zhou, H., Oliphant, M. U. J., Zhang, X., Simon, L. M., et al. (2017). EMT cells increase breast cancer metastasis via paracrine GLI activation in neighbouring tumour cells. Nature Communications, 8, 15773. doi:10.1038/ncomms15773

Nie, Y., Huang, H., Guo, M., Chen, J., Wu, W., et al. (2019). Breast Phyllodes Tumors Recruit and Repolarize Tumor-Associated Macrophages via Secreting CCL5 to Promote Malignant Progression, Which Can Be Inhibited by CCR5 Inhibition Therapy. Clin Cancer Res, 25, 3873-3886. doi:10.1158/1078-0432.ccr-18-3421

Noubissi, F. K., Harkness, T., Alexander, C. M., \& Ogle, B. M. (2015). Apoptosis-induced cancer cell fusion: a mechanism of breast cancer metastasis. Faseb j, 29, 4036-4045. doi:10.1096/fj.15-271098

Oliveira, M. N., Pillat, M. M., Motaln, H., Ulrich, H., \& Lah, T. T. (2018). Kinin-B1 receptor stimulation promotes invasion and is involved in cell-cell interaction of co-cultured glioblastoma and mesenchymal stem cells. Scientific Reports, 8, 1-16.

Pawelek, J. M. (2000). Tumour cell hybridization and metastasis revisited. Melanoma Research, 10, 507-514. doi:10.1097/00008390-200012000-00001

Pawelek, J. M. (2005). Tumour-cell fusion as a source of myeloid traits in cancer. The Lancet Oncology, 6, 988-993. doi:https://doi.org/10.1016/S1470-2045(05)70466-6

Pawelek, J. M., \& Chakraborty, A. K. (2008). Chapter 10 The Cancer Cell-Leukocyte Fusion Theory of Metastasis. In Advances in Cancer Research (Vol. 101, pp. 397-444): Academic Press.

Raslova, H., Kauffmann, A., Sekkaï, D., Ripoche, H., Larbret, F., et al. (2007). Interrelation between polyploidization and megakaryocyte differentiation: a gene profiling approach. Blood, 109, 3225-3234. doi:10.1182/blood-2006-07-037838

Sahoo, S., Mishra, A., Kaur, H., Hari, K., Muralidharan, S., et al. (2021). A mechanistic model captures the emergence and implications of non-genetic heterogeneity and reversible drug resistance in ER+ breast cancer cells. NAR Cancer, 3, zcab027. doi:10.1093/narcan/zcab027

Sahoo, S., Nayak, S. P., Hari, K., Purkait, P., Mandal, S., et al. (2021). Immunosuppressive traits of the hybrid epithelial/mesenchymal phenotype. bioRxiv, 2021.2006.2021.449285. doi:10.1101/2021.06.21.449285

Saito, T., Kasamatsu, A., Ogawara, K., Miyamoto, I., Saito, K., et al. (2015). Semaphorin7A Promotion of Tumoral Growth and Metastasis in Human Oral Cancer by Regulation of 
G1 Cell Cycle and Matrix Metalloproteases: Possible Contribution to Tumoral Angiogenesis. PLoS One, 10, e0137923. doi:10.1371/journal.pone.0137923

Searles, S. C., Santosa, E. K., \& Bui, J. D. (2018). Cell-cell fusion as a mechanism of DNA exchange in cancer. Oncotarget, 9, 6156-6173. doi:10.18632/oncotarget.23715

Shabo, I., Midtbö, K., Andersson, H., Åkerlund, E., Olsson, H., et al. (2015). Macrophage traits in cancer cells are induced by macrophage-cancer cell fusion and cannot be explained by cellular interaction. BMC Cancer, 15, 922. doi:10.1186/s12885-015-1935-0

Sidebottom, E., \& Clark, S. R. (1983). Cell fusion segregates progressive growth from metastasis. British Journal of Cancer, 47, 399-405. doi:10.1038/bjc.1983.60

Sieler, M., Weiler, J., \& Dittmar, T. (2021). Cell-Cell Fusion and the Roads to Novel Properties of Tumor Hybrid Cells. Cells, 10, 1465.

Soltantoyeh, T., Akbari, B., Karimi, A., Mahmoodi Chalbatani, G., Ghahri-Saremi, N., et al. (2021). Chimeric Antigen Receptor (CAR) T Cell Therapy for Metastatic Melanoma: Challenges and Road Ahead. Cells, 10. doi:10.3390/cells10061450

Suetsugu, A., \& Hoffman, R. M. (2021). Color-Coded Imaging of Cancer and Stromal-Cell Interaction in the Pancreatic-Cancer Tumor Microenvironment (TME). In Mouse Genetics (pp. 99-111): Springer.

Truffi, M., Sorrentino, L., \& Corsi, F. (2020). Fibroblasts in the Tumor Microenvironment. Adv Exp Med Biol, 1234, 15-29. doi:10.1007/978-3-030-37184-5_2

Tsuji, T., Ibaragi, S., Shima, K., Hu, M. G., Katsurano, M., et al. (2008). Epithelialmesenchymal transition induced by growth suppressor $\mathrm{p} 12 \mathrm{CDK} 2-\mathrm{AP} 1$ promotes tumor cell local invasion but suppresses distant colony growth. Cancer Research, 68, 10377 10386. doi:10.1158/0008-5472.can-08-1444

Uygur, B., Leikina, E., Melikov, K., Villasmil, R., Verma, S. K., et al. (2019). Interactions with muscle cells boost fusion, stemness, and drug resistance of prostate cancer cells. Molecular Cancer Research, 17, 806-820.

van Zijl, F., Krupitza, G., \& Mikulits, W. (2011). Initial steps of metastasis: cell invasion and endothelial transmigration. Mutat Res, 728, 23-34. doi:10.1016/j.mrrev.2011.05.002

Vorträge und Aufsätze über entwickelungsmechanik der Organismen. (1905). Springer-Verlag.

Vucicevic Boras, V., Fucic, A., Virag, M., Gabric, D., Blivajs, I., et al. (2018). Significance of stroma in biology of oral squamous cell carcinoma. Tumori, 104, 9-14. doi:10.5301/tj.5000673

Wang, R., Sun, X., Wang, C. Y., Hu, P., Chu, C. Y., et al. (2012). Spontaneous cancer-stromal cell fusion as a mechanism of prostate cancer androgen-independent progression. PLoS One, 7, e42653. doi:10.1371/journal.pone.0042653

Wang, Y., Fan, H., Zhou, B., Ju, Z., Yu, L., et al. (2012). Fusion of human umbilical cord mesenchymal stem cells with esophageal carcinoma cells inhibits the tumorigenicity of esophageal carcinoma cells. International Journal of Oncology, 40, 370-377. doi:10.3892/ijo.2011.1232

Wei, H. J., Nickoloff, J. A., Chen, W. H., Liu, H. Y., Lo, W. C., et al. (2014). FOXF1 mediates mesenchymal stem cell fusion-induced reprogramming of lung cancer cells. Oncotarget, 5, 9514-9529. doi:10.18632/oncotarget.2413

Weiler, J., Mohr, M., Zänker, K. S., \& Dittmar, T. (2018). Matrix metalloproteinase-9 (MMP9) is involved in the TNF- $\alpha$-induced fusion of human M13SV1-Cre breast epithelial cells and human MDA-MB-435-pFDR1 cancer cells. Cell Commun Signal, 16, 14. doi:10.1186/s12964-018-0226-1

Xu, M. H., Gao, X., Luo, D., Zhou, X. D., Xiong, W., et al. (2014). EMT and acquisition of stem cell-like properties are involved in spontaneous formation of tumorigenic hybrids between lung cancer and bone marrow-derived mesenchymal stem cells. PloS One, 9, e87893. doi:10.1371/journal.pone.0087893 
Xue, J., Zhu, Y., Sun, Z., Ji, R., Zhang, X., et al. (2015). Tumorigenic hybrids between mesenchymal stem cells and gastric cancer cells enhanced cancer proliferation, migration and stemness. BMC Cancer, 15, 793. doi:10.1186/s12885-015-1780-1

Yang, H., Wang, B., Wang, T., Xu, L., He, C., et al. (2014). Toll-like receptor 4 prompts human breast cancer cells invasiveness via lipopolysaccharide stimulation and is overexpressed in patients with lymph node metastasis. PloS One, 9, e109980. doi:10.1371/journal.pone.0109980

Yilmaz, Y., Lazova, R., Qumsiyeh, M., Cooper, D., \& Pawelek, J. (2005). Donor Y chromosome in renal carcinoma cells of a female BMT recipient: visualization of putative BMT-tumor hybrids by FISH. Bone Marrow Transplant, 35, 1021-1024. doi:10.1038/sj.bmt.1704939

Yin, M., Li, X., Tan, S., Zhou, H. J., Ji, W., et al. (2016). Tumor-associated macrophages drive spheroid formation during early transcoelomic metastasis of ovarian cancer. $J$ Clin Invest, 126, 4157-4173. doi:10.1172/jci87252

Zhang, L. N., Huang, Y. H., \& Zhao, L. (2019). Fusion of macrophages promotes breast cancer cell proliferation, migration and invasion through activating epithelial-mesenchymal transition and Wnt/ß-catenin signaling pathway. Arch Biochem Biophys, 676, 108137. doi:10.1016/j.abb.2019.108137

Zhang, L. N., Kong, C. F., Zhao, D., Cong, X. L., Wang, S. S., et al. (2019). Fusion with mesenchymal stem cells differentially affects tumorigenic and metastatic abilities of lung cancer cells. Journal of Cellular Physiology, 234, 3570-3582. doi:10.1002/jcp.27011

Zhang, P., Wei, Y., Wang, L., Debeb, B. G., Yuan, Y., et al. (2014). ATM-mediated stabilization of ZEB1 promotes DNA damage response and radioresistance through CHK1. Nat Cell Biol, 16, 864-875. doi:10.1038/ncb3013

Zhang, Y., Luo, W., Wang, Y., Liu, Y., \& Zheng, L. (2014). Purified dendritic cell-tumor fusion hybrids supplemented with non-adherent dendritic cells fraction are superior activators of antitumor immunity. PloS One, 9, e86772.

Zheng, X., Carstens, J. L., Kim, J., Scheible, M., Kaye, J., et al. (2015). Epithelial-tomesenchymal transition is dispensable for metastasis but induces chemoresistance in pancreatic cancer. Nature, 527, 525-530. doi:10.1038/nature16064

Zhu, C., Mustafa, D., Zheng, P. P., van der Weiden, M., Sacchetti, A., et al. (2017). Activation of CECR1 in M2-like TAMs promotes paracrine stimulation-mediated glial tumor progression. Neuro Oncol, 19, 648-659. doi:10.1093/neuonc/now251

Zhu, H., Peng, B., Klausen, C., Yi, Y., Li, Y., et al. (2020). NPFF increases fusogenic proteins syncytin 1 and syncytin 2 via GCM1 in first trimester primary human cytotrophoblast cells. Faseb j, 34, 9419-9432. doi:10.1096/fj.201902978R 
Table 1: Summary of reports showing the fusion of different cancer cells with immune and stromal cells and their impact on EMT, CSCs and metastasis

\begin{tabular}{|c|c|c|c|c|c|c|}
\hline Cancer cell & Stromal cell & Fusion & Migration/invasion assay & $\begin{array}{r}\text { CSC ch } \\
\text { (Surfa } \\
\text { Function }\end{array}$ & $\begin{array}{l}\text { cterization } \\
\text { markers/ } \\
\text { assay done) }\end{array}$ & Reference \\
\hline $\begin{array}{l}\text { Human H460 and } \\
\text { A549 NSCLC lines }\end{array}$ & Monocytes & $<4 \%$ & Transwell migration & $\begin{array}{l}\text { Nanog, Oct } 3 / 4, \text { KLF } 4, \\
\text { Sox } 2 \text {, MYC }\end{array}$ & $\begin{array}{l}\text { Formation of spheroid } \\
\text { aggregates }\end{array}$ & $\begin{array}{l}\text { (Aguirre et } \\
\text { al., 2020) }\end{array}$ \\
\hline $\begin{array}{l}\text { T47D human } \\
\text { breast cancer cells }\end{array}$ & $\begin{array}{l}\text { Human } \\
\text { mesenchymal } \\
\text { stromal cells }\end{array}$ & & Timelapse microscopy & & & $\begin{array}{l}\text { (Chitwood } \\
\text { et al., 2018) }\end{array}$ \\
\hline $\begin{array}{l}\text { Peripheral blood } \\
\text { from PDAC } \\
\text { patients, MC } 38 \\
\text { mouse intestinal } \\
\text { epithelial cancer } \\
\text { cells }\end{array}$ & Macrophages & $0.48 \%$ & $\begin{array}{l}\text { Boyden chamber invasion } \\
\text { assay }\end{array}$ & & & $\begin{array}{l}\text { (Gast et al., } \\
\text { 2018) }\end{array}$ \\
\hline $\begin{array}{l}\text { IB105/106 sarcoma } \\
\text { cell lines }\end{array}$ & $\begin{array}{l}\text { IMR90 } \\
\text { fibroblasts }\end{array}$ & & $\begin{array}{l}\text { Scratch assay, Boyden } \\
\text { chamber invasion assay }\end{array}$ & & $\begin{array}{l}\text { Soft agar colony } \\
\text { formation assay }\end{array}$ & $\begin{array}{l}\text { (Lartigue et } \\
\text { al., 2020) }\end{array}$ \\
\hline $\begin{array}{l}\text { Human MDA-MB- } \\
231 \text { breast cancer } \\
\text { cells }\end{array}$ & $\begin{array}{l}\text { Mesenchymal } \\
\text { stroma/stem cells }\end{array}$ & & & $\begin{array}{l}\text { CD29, CD44, CD73, } \\
\text { CD90, CD105, CD146, } \\
\text { CD166 }\end{array}$ & In vivo tumorigenicity & $\begin{array}{l}\text { (C. Melzer } \\
\text { et al., 2018) }\end{array}$ \\
\hline $\begin{array}{l}\text { Human MDA-MB- } \\
231 \text { breast cancer } \\
\text { cells }\end{array}$ & $\begin{array}{l}\text { Mesenchymal } \\
\text { stroma/stem cells }\end{array}$ & $\begin{array}{l}0.015- \\
0.18 \%\end{array}$ & & $\begin{array}{l}\text { CD } 44, \text { CD73, CD90, } \\
\text { CD105 }\end{array}$ & In vivo tumorigenicity & $\begin{array}{l}\text { (C. Melzer } \\
\text { et al., 2019) }\end{array}$ \\
\hline $\begin{array}{l}\text { IMR90- E6E7 } \\
\text { HRAS }^{\mathrm{G} 12 \mathrm{~V}}\end{array}$ & $\begin{array}{l}\text { IMR90- E6E7 } \\
\text { fibroblasts }\end{array}$ & $2 \%$ & Scratch assay & $\begin{array}{l}\text { ALDH, NANOG } \\
\text { and OCT4 }\end{array}$ & Sphere formation assay & $\begin{array}{l}\text { (Merle et } \\
\text { al., 2021) }\end{array}$ \\
\hline
\end{tabular}




\section{Figures}

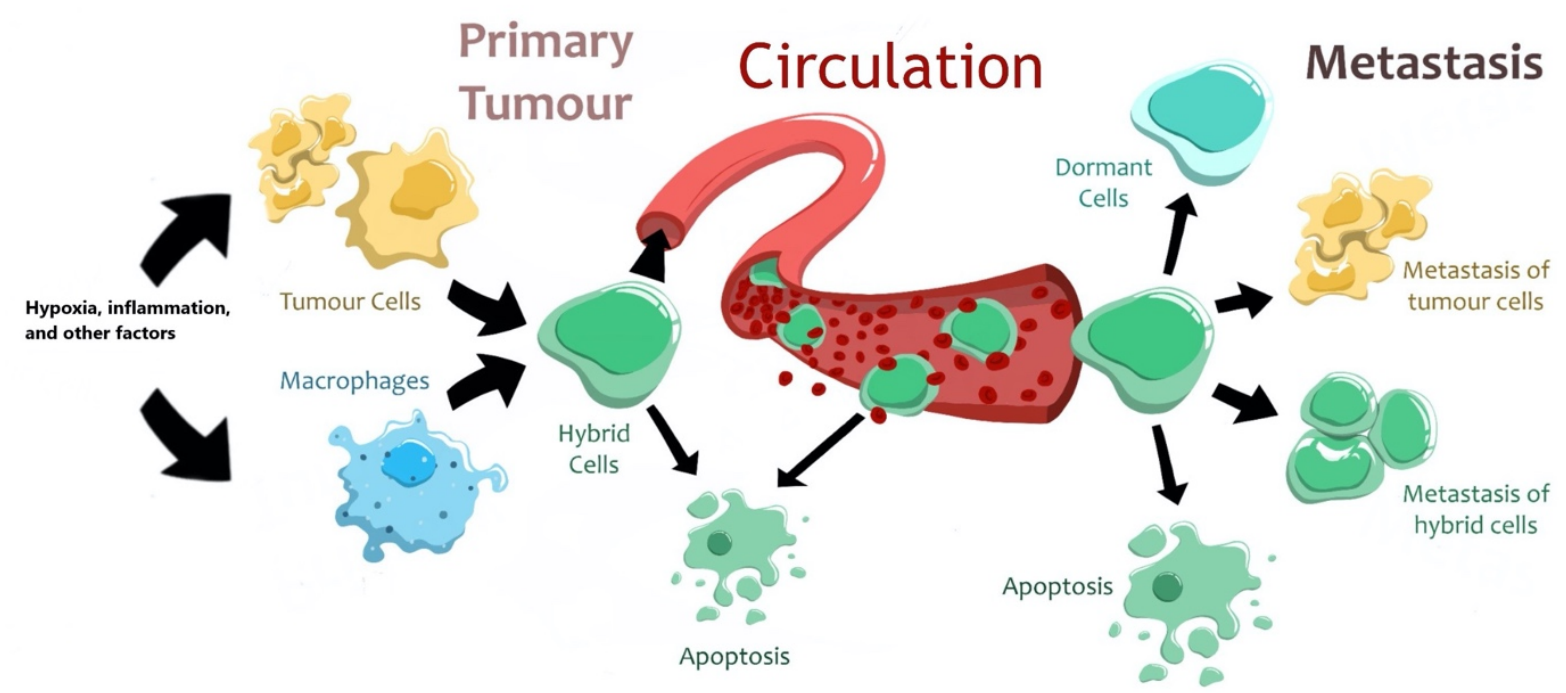

Fig 1: The proposed life cycle of a tumor-macrophage fusion cell. Tumor-macrophage fusion cell is formed via fusion of tumor cell from the primary tumor with a macrophage that is triggered by hypoxia, inflammation, or other factors. This hybrid cell can either undergo apoptosis or enter circulation. Then, this cell travels to a secondary site or dies via apoptosis in the bloodstream. After reaching the target organ, the hybrid cell can form metastasis directly or via the return to the tumor state by getting rid of the macrophage material.

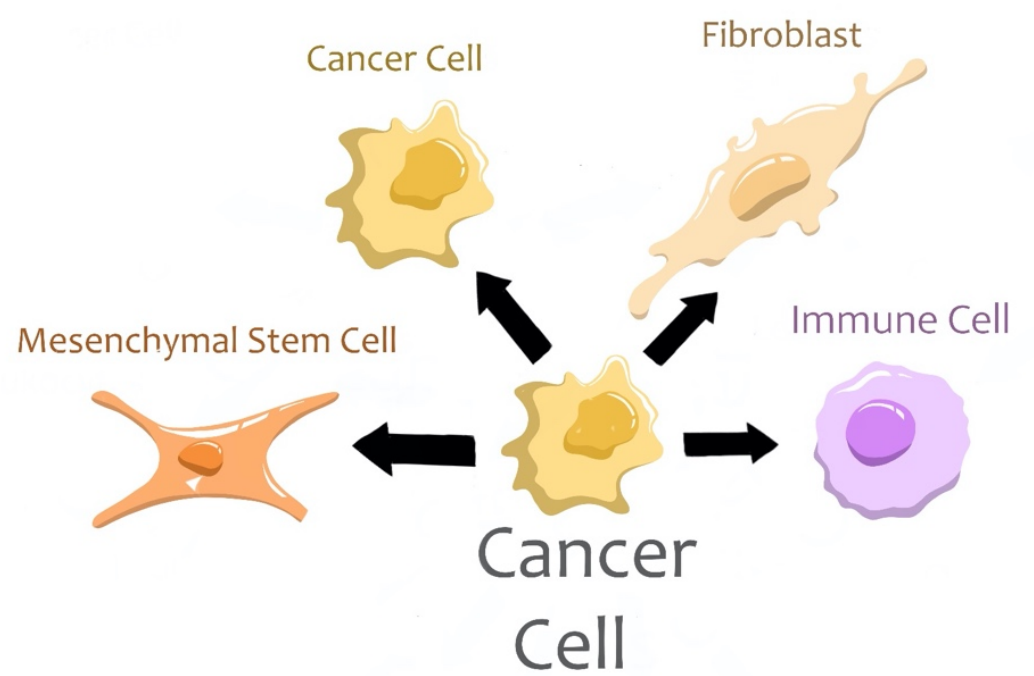

Fig 2: Types of tumor hybrid cells. 


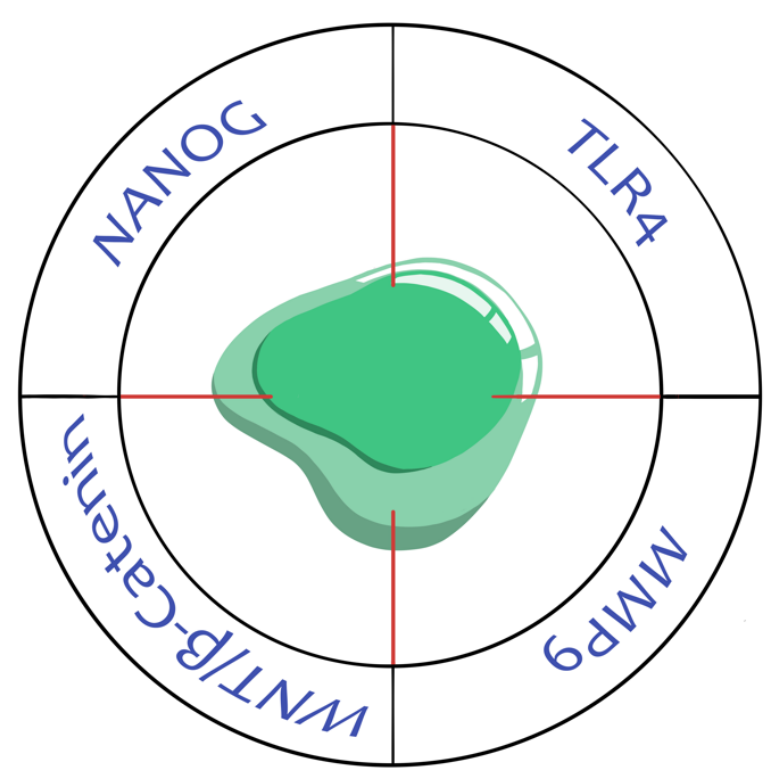

Fig 3: Potential therapeutic targets in tumor hybrid cells. TLR4, toll-like receptor 4; MMP9, matrix metalloproteinase 9; Wnt/ $\beta$-catenin, signaling pathway; Nanog, transcription factor. 